\title{
International Journal of
}

\section{Information Engineering and Electronic Business}

Kol.7

No. 1 Jan. 2015

\section{IJIEEB Voı.7}

Http:// www.mecs-press .org

Vol. 7 No. 1 January 2015
Modern Education

and Computer Science PRESS 


\section{International Journal of Information Engineering and Electronic Business (IJIEEB)}

ISSN Print: 2074-9023, ISSN Online: 2074-9031

Volume 7, Number 1, January 2015

\section{Contents}

\section{REGULAR PAPERS}

Compromise Hypersphere for Multi-Criteria Dynamic Programming

Sebastian Sitarz

A Web 2.0 Supported Business Process Management Environment for Collaborative Research

Asli Sencer, Meltem Ozturan, Hande Kimiloglu

Effective Networking Model for Efficient Implementation of E-Governance: A Case Study of Nigeria

Lauretta O. Osho, Muhammad B. Abdullahi, Oluwafemi Osho, John K. Alhassan

Rank University Websites Using Fuzzy AHP and Fuzzy TOPSIS Approach on Usability

Renuka Nagpal, Deepti Mehrotra, Pradeep Kumar Bhatia, Arun Sharma

Proposal of Enhanced Extreme Programming Model

M. Rizwan Jameel Qureshi, Jacob S. Ikram

Enable Better and Timelier Decision-Making Using Real-Time Business Intelligence System

Darshan M. Tank

A Content Assist based Approach for Providing Rationale of Method Change for Object Oriented

Programming

Amit S. Ami, Shariful Islam

OpenMP Dual Population Genetic Algorithm for Solving Constrained Optimization Problems

A. J. Umbarkar, M. S. Joshi, P. D. Sheth 